\title{
On Fractional Variational Problems which Admit Local Transformations
}

\author{
Agnieszka B. Malinowska \\ a.malinowska@pb.edu.pl \\ Faculty of Computer Science, Białystok University of Technology, \\ 15-351 Białystok, Poland
}

\begin{abstract}
We extend the second Noether theorem to fractional variational problems which are invariant under infinitesimal transformations that depend upon $r$ arbitrary functions and their fractional derivatives in the sense of Caputo. Our main result is illustrated using the fractional Lagrangian density of the electromagnetic field.
\end{abstract}

Keywords: Lagrangian systems; fractional calculus; gauge symmetries; second Noether's theorem; electromagnetic field.

MSC: 49K05, 49S05, 26A33

PACS: 04.20.Fy, 45.10.Db, 45.10.Hj

\section{Introduction}

In 1918 Emmy Noether published a paper that is now famous for Noether's theorem. But in fact Noether proved two theorems in the 1918 paper. The first theorem explains the correspondence between conserved quantities and continuous symmetry transformations that depend on constant parameters. Such transformations are global transformations. Familiar examples from classical mechanics include the connections between: spatial translations and conservation of linear momentum; spatial rotations and conservation of angular momentum; and time translations and conservation of energy (Gelfand and Fomin (2000); Logan (1977)). The second theorem, less well known, guarantees syzygies between the Euler-Lagrange equations for a variational problem which is invariant under transformations that depend on arbitrary functions and their derivatives. Such transformations are local transformations. The statement of the theorem for this case is very general and, aside from its application to general relativity, it applies in a wide variety of other cases. For example, quantum chromodynamics and other gauge field theories are theories to which it applies. From the second theorem, one has identities between Lagrange expressions and their derivatives. These identities Noether called "dependencies". For example, the Bianchi identities, in the general theory of relativity, are examples of such "dependencies". In electrodynamics, if the Lagrangian represents a charged particle interacting with a electromagnetic field, one finds that it is invariant under the combined action of the so called gauge transformation of the first kind 
on the charged particle field, and a gauge transformation of the second kind on the electromagnetic field. As a result of this invariance it follows, from second Noether's theorem, the conservation of charge. For a complete history of Noether's two theorems on variational symmetries see Brading (2002); Kosmann-Schwarzbach (2010); and also Carinena et al. (2005); Hydon and Mansfield (2011); Logan (1974); Torres (2003) for some other generalizations.

Fractional calculus is a discipline that studies integrals and derivatives of non-integer (real or complex) order (Kilbas et al. (2006); Klimek (2009); Podlubny (1999); Samko et al. $(1993)$ ). The field was born in 1695 and became an ongoing topic with many well-known mathematicians contributing to its theory (see Tenreiro Machado et al. (2010) for a review). Fractional derivatives are nonlocal operators and are historically applied in the study of nonlocal or time dependent processes. The first and well established application of fractional calculus in Physics was in the framework of anomalous diffusion, which are related to features observed in many physical systems, e.g. in dispersive transport in amorphous semiconductor, liquid crystals, polymers, proteins, etc. Electromagnetic equations (Baleanu et al. (2009)) and field theories (Almeida et al. (2010); Cresson (2007); Herrmann (2008); Tarasov (2008)) have been also recently considered in the context of fractional calculus. The subject is nowadays very active due to its many applications in mechanics, chemistry, biology, economics, and control theory (see Baleanu et al. (2011); Fonseca Ferreira et al. (2008); Hilfer (2000); Tenreiro Machado et al. (2011) for a review).

One of the most remarkable applications of fractional calculus is in the context of classical mechanics. Riewe (1996, 1997) showed that a Lagrangian involving fractional time derivatives leads to an equation of motion with nonconservative forces such as friction. It is a remarkable result since frictional and nonconservative forces are beyond the usual macroscopic variational treatment, and consequently, beyond the most advanced methods of classical mechanics. Riewe generalized the usual variational calculus, by considering Lagrangians that dependent on fractional derivatives, in order to deal with nonconservative forces. Recently, several approaches have been developed to generalize the least action principle and the Euler-Lagrange equations to include fractional derivatives. Investigations cover problems depending on the Caputo fractional derivatives (Agrawal (2007); Almeida et al. (2012); Baleanu and Agrawal (2006); Baleanu (2008); Malinowska and Torres (2010, 2011); Tarasov (2006)), the Riemann-Liouville fractional derivatives (Almeida and Torres (2009); Atanacković et al. (2008); Baleanu and Avkar (2004); Baleanu and Muslih (2005); Herzallah and Baleanu (2011)) and others (Agrawal et al. (2011); El-Nabulsi and Torres (2007); El-Nabulsi (2011); Jumarie (2007); Malinowska (2012)).

Noether's first theorems have been extended to fractional variational problems using several approaches (Atanacković et al. (2009); Cresson (2007); Frederico and Torres (2007, 2008, 2010)). To the best of our knowledge, no second Noether type theorem is available for the fractional setting. Such a generalization is the aim of this paper. We prove the fractional second Noether theorem for one and multiple dimensional Lagrangians, and we show how our results can be applied for fractional electromagnetic field. We trust that this paper will open several new directions of research and applications. Although we choose the Caputo calculus, our results are general and can be straightforward generalized to other fractional calculus, like the Riemann-Liouville fractional calculus and others approaches.

The paper is organized in the following way. In Section 2 we recall the notion of fractional derivatives and their basic properties, that are needed in the sequel. The intended fractional second Noether type theorem is formulated and proved in Section 3, for single (Subsection 3.1) and multiple (Subsection 3.2) integral problems. Our main result is illustrated in Section 4 
using the fractional Lagrangian density of the electromagnetic field.

\section{Fractional Derivatives}

In this section we review the necessary definitions and facts from the fractional calculus. For more on the subject we refer the reader to (Kilbas et al. (2006); Klimek (2009); Podlubny (1999); Samko et al. (1993)).

Let $\alpha \in \mathbb{R}$ and $0<\alpha<1, f \in L_{1}([a, b], \mathbb{R})$. By the left Riemann-Liouville fractional integral of $f$ on the interval $[a, b]$ we mean a function ${ }_{a} I_{x}^{\alpha} f$ defined by:

$$
{ }_{a} I_{x}^{\alpha} f(x)=\frac{1}{\Gamma(\alpha)} \int_{a}^{x}(x-t)^{\alpha-1} f(t) d t, \quad x \in[a, b] \text { a.e. }
$$

By the right Riemann-Liouville fractional integral of $f$ on the interval $[a, b]$ we mean a function ${ }_{x} I_{b}^{\alpha} f$ defined by:

$$
{ }_{x} I_{b}^{\alpha} f(x)=\frac{1}{\Gamma(\alpha)} \int_{x}^{b}(t-x)^{\alpha-1} f(t) d t, \quad x \in[a, b] \text { a.e. }
$$

$\Gamma(\cdot)$ represents the Gamma function. For $\alpha=0$, we set ${ }_{a} I_{x}^{0} f={ }_{x} I_{b}^{0} f:=I f$, the identity operator.

If the function ${ }_{a} I_{x}^{\alpha} f$ is absolutely continuous on the interval $[a, b]$, then the left RiemannLiouville fractional derivative is given by:

$$
{ }_{a} D_{x}^{\alpha} f(x)=\frac{1}{\Gamma(1-\alpha)} \frac{d}{d x} \int_{a}^{x}(x-t)^{-\alpha} f(t) d t=\frac{d}{d x}{ }_{a} I_{x}^{1-\alpha} f(x) .
$$

If the function ${ }_{x} I_{b}^{1-\alpha} f$ is absolutely continuous on the interval $[a, b]$, then the right RiemannLiouville fractional derivative is given by:

$$
{ }_{x} D_{b}^{\alpha} f(x)=\frac{-1}{\Gamma(1-\alpha)} \frac{d}{d x} \int_{x}^{b}(t-x)^{-\alpha} f(t) d t=\left(-\frac{d}{d x}\right){ }_{x} I_{b}^{1-\alpha} f(x) .
$$

Let $f \in A C([a, b], \mathbb{R})$. By the left Caputo fractional derivative of $f$ on the interval $[a, b]$ we mean a function ${ }_{a}^{C} D_{x}^{\alpha} f$ defined by:

$$
{ }_{a}^{C} D_{x}^{\alpha} f(x)=\frac{1}{\Gamma(1-\alpha)} \int_{a}^{x}(x-t)^{-\alpha} \frac{d}{d t} f(t) d t={ }_{a} I_{x}^{1-\alpha} \frac{d}{d x} f(x),
$$

and by the right Caputo fractional derivative of $f$ on the interval $[a, b]$ we mean a function ${ }_{a}^{C} D_{x}^{\alpha} f$ defined by:

$$
{ }_{x}^{C} D_{b}^{\alpha} f(x)=\frac{-1}{\Gamma(1-\alpha)} \int_{x}^{b}(t-x)^{-\alpha} \frac{d}{d t} f(t) d t={ }_{x} I_{b}^{1-\alpha}\left(-\frac{d}{d x}\right) f(x) .
$$

Remark 1. Observe that if $\alpha$ goes to 1 , then under suitable assumptions operators ${ }_{a} D_{x}^{\alpha}$ and ${ }_{a}^{C} D_{x}^{\alpha}$ can be replaced with $\frac{d}{d x}$, and operators ${ }_{x} D_{b}^{\alpha}$ and ${ }_{x}^{C} D_{b}^{\alpha}$ can be replaced with $-\frac{d}{d x}$ (see Podlubny (1999)).

The operators (1) -(6) are obviously linear. Below we present the rules of fractional integration by parts for Riemann-Liouville fractional integral and Caputo fractional derivatives which are particularly useful for our purposes. 
Lemma 2.1 (Love and Young (1938)). Let $0<\alpha<1, p \geq 1, q \geq 1$, and $1 / p+1 / q \leq 1+\alpha$. If $g \in L_{p}([a, b])$ and $f \in L_{q}([a, b])$, then

$$
\int_{a}^{b} g(x)_{a} I_{x}^{\alpha} f(x) d x=\int_{a}^{b} f(x)_{x} I_{b}^{\alpha} g(x) d x .
$$

Lemma 2.2. [cf. Klimek (2009)] Let $0<\alpha<1$. If $f, g \in A C([a, b])$, then

$$
\begin{aligned}
& \int_{a}^{b} g(x){ }_{a}^{C} D_{x}^{\alpha} f(x) d x=\left.f(x)_{x} I_{b}^{1-\alpha} g(x)\right|_{x=a} ^{x=b}+\int_{a}^{b} f(x)_{x} D_{b}^{\alpha} g(x) d x, \\
& \int_{a}^{b} g(x)_{x}^{C} D_{b}^{\alpha} f(x) d x=-\left.f(x)_{a} I_{x}^{1-\alpha} g(x)\right|_{x=a} ^{x=b}+\int_{a}^{b} f(x)_{a} D_{x}^{\alpha} g(x) d x .
\end{aligned}
$$

Proof. Formulas could be derived using equations (3)-(6), the identity (7) and performing standard integration by parts.

Partial fractional integrals and derivatives are a natural generalization of the corresponding one-dimensional fractional integrals and derivatives, being taken with respect to one or several variables. For $\left(x_{1}, \ldots, x_{n}\right),\left(\alpha_{1}, \ldots, \alpha_{n}\right)$, where $0<\alpha_{i}<1, i=1, \ldots, n$ and $\left[a_{1}, b_{1}\right] \times \ldots \times\left[a_{n}, b_{n}\right]$, the partial Riemann-Liouville fractional integrals of order $\alpha_{k}$ with respect to $x_{k}$ are defined by

$$
\begin{aligned}
& a_{k} I_{x_{k}}^{\alpha_{k}} f\left(x_{1}, \ldots, x_{n}\right)=\frac{1}{\Gamma\left(\alpha_{k}\right)} \int_{a_{k}}^{x_{k}}\left(x_{k}-t_{k}\right)^{\alpha_{k}-1} f\left(x_{1}, \ldots, x_{k-1}, t_{k}, x_{k+1}, \ldots, x_{n}\right) d t_{k}, \quad x_{k}>a_{k}, \\
& x_{k} I_{b_{k}}^{\alpha_{k}} f\left(x_{1}, \ldots, x_{n}\right)=\frac{1}{\Gamma\left(\alpha_{k}\right)} \int_{x_{k}}^{b_{k}}\left(t_{k}-x_{k}\right)^{\alpha_{k}-1} f\left(x_{1}, \ldots, x_{k-1}, t_{k}, x_{k+1}, \ldots, x_{n}\right) d t_{k}, \quad x_{k}<b_{k} .
\end{aligned}
$$

Partial Riemann-Liouville and Caputo derivatives are defined by:

$$
\begin{aligned}
a_{k} D_{x_{k}}^{\alpha_{k}} f\left(x_{1}, \ldots, x_{n}\right) & =\frac{1}{\Gamma\left(1-\alpha_{k}\right)} \frac{\partial}{\partial x_{k}} \int_{a_{k}}^{x_{k}}\left(x_{k}-t_{k}\right)^{-\alpha_{k}} f\left(x_{1}, \ldots, x_{k-1}, t_{k}, x_{k+1}, \ldots, x_{n}\right) d t_{k}, \\
x_{k} D_{b_{k}}^{\alpha_{k}} f\left(x_{1}, \ldots, x_{n}\right) & =-\frac{1}{\Gamma\left(1-\alpha_{k}\right)} \frac{\partial}{\partial x_{k}} \int_{x_{k}}^{b_{k}}\left(t_{k}-x_{k}\right)^{-\alpha_{k}} f\left(x_{1}, \ldots, x_{k-1}, t_{k}, x_{k+1}, \ldots, x_{n}\right) d t_{k}, \\
{ }_{a_{k}}^{C} D_{x_{k}}^{\alpha_{k}} f\left(x_{1}, \ldots, x_{n}\right) & =\frac{1}{\Gamma\left(1-\alpha_{k}\right)} \int_{a_{k}}^{x_{k}}\left(x_{k}-t_{k}\right)^{-\alpha_{k}} \frac{\partial}{\partial t_{k}} f\left(x_{1}, \ldots, x_{k-1}, t_{k}, x_{k+1}, \ldots, x_{n}\right) d t_{k}, \\
{ }_{x_{k}}^{C} D_{b_{k}}^{\alpha_{k}} f\left(x_{1}, \ldots, x_{n}\right) & =-\frac{1}{\Gamma\left(1-\alpha_{k}\right)} \int_{x_{k}}^{b_{k}}\left(t_{k}-x_{k}\right)^{-\alpha_{k}} \frac{\partial}{\partial t_{k}} f\left(x_{1}, \ldots, x_{k-1}, t_{k}, x_{k+1}, \ldots, x_{n}\right) d t_{k} .
\end{aligned}
$$

\section{Main Results}

In this Section we formulate and prove the fractional second Noether type theorem, for single (Subsection 3.1) and multiple (Subsection 3.2) integral problems. 


\subsection{Single Integral Case}

Consider a system characterized by a set of functions

$$
x^{i}(t), \quad i=1, \ldots, n,
$$

depending on time $t$. We can simplify the notation by interpreting (91) as a vector function $x=\left(x^{1}, \ldots, x^{n}\right)$. Define the action functional in the form

$$
\mathcal{J}(x)=\int_{a}^{b} L\left(t, x(t),{ }_{a}^{C} D_{t}^{\alpha} x(t)\right) d t
$$

where:

(i) ${ }_{a}^{C} D_{t}^{\alpha} x(t):=\left({ }_{a}^{C} D_{t}^{\alpha_{1}} x^{1}(t), \ldots,{ }_{a}^{C} D_{t}^{\alpha_{n}} x^{n}(t)\right), 0<\alpha_{i} \leq 1, i=1, \ldots, n$;

(ii) $x \in C^{1}\left([a, b], \mathbb{R}^{n}\right)$;

(iii) $L \in C^{1}\left([a, b] \times \mathbb{R}^{2 n}, \mathbb{R}\right)$;

(iv) $t \rightarrow \frac{\partial L}{\partial_{a}^{C} D_{t}^{\alpha_{k}} x^{k}} \in A C([a, b])$ for every $x \in C^{1}\left([a, b], \mathbb{R}^{n}\right), k=1, \ldots, n$.

We define the admissible set of functions $A([a, b])$ by

$$
A([a, b]):=\left\{x \in C^{1}\left([a, b], \mathbb{R}^{n}\right): x(a)=x_{a}, x(b)=x_{b}, x_{a}, x_{b} \in \mathbb{R}^{n}\right\} .
$$

Theorem 3.1 (Agrawal (2007)). A necessary condition for the function $x \in A([a, b])$ to provide an extremum for the functional (10) is that its components satisfy the $n$ fractional equations

$$
\frac{\partial L}{\partial x^{k}}+{ }_{t} D_{b}^{\alpha_{k}} \frac{\partial L}{\partial_{a}^{C} D_{t}^{\alpha_{k}} x^{k}}=0, \quad k=1, \ldots, n
$$

for $t \in[a, b]$.

Define

$$
E_{k}^{f}(L):=\frac{\partial L}{\partial x^{k}}+{ }_{t} D_{b}^{\alpha_{k}} \frac{\partial L}{\partial_{a}^{C} D_{t}^{\alpha_{k}} x^{k}}
$$

We shall call $E_{k}^{f}(L)$ the fractional Lagrange expressions.

The invariance transformations that we shall consider are infinitesimal transformations that depend upon arbitrary functions and their fractional derivatives in the sense of Caputo. Let

$$
\left\{\begin{array}{l}
\bar{t}=t, \\
\bar{x}^{k}(t)=x^{k}(t)+T^{k 1}\left(p_{1}(t)\right)+\cdots+T^{k r}\left(p_{r}(t)\right), \quad k=1, \ldots, n,
\end{array}\right.
$$

where $T^{k s}$ are linear fractional differential operators and $p_{s}, s=1, \ldots, r$ are $r$ arbitrary, independent $C^{1}$ functions defined on $[a, b]$. Then, we consider four types of fractional differential operators:

I. Operator of the first kind

$$
\begin{aligned}
& \quad T^{k s}=T_{1}^{k s}:=a_{0}^{k s}(t)+a_{1}^{k s}(t)_{a}^{C} D_{t}^{\beta_{k s 1}}+\cdots+a_{l}^{k s}(t)_{a}^{C} D_{t}^{\beta_{k s l}}, \quad 0<\beta_{k s i} \leq 1, \\
& \text { and }{ }_{a}^{C} D_{t}^{\beta_{k s i}} p_{s} \in C^{1}([a, b]), a_{i}^{k s} \in C^{1}([a, b], \mathbb{R}), s=1, \ldots, r, i=1, \ldots, l .
\end{aligned}
$$


II. Operator of the second kind

$$
T^{k s}=T_{2}^{k s}:=a_{0}^{k s}(t)+a_{1}^{k s}(t)_{t}^{C} D_{b}^{\beta_{k s 1}}+\cdots+a_{l}^{k s}(t)_{t}^{C} D_{b}^{\beta_{k s l}}, \quad 0<\beta_{k s i} \leq 1,
$$

and ${ }_{t}^{C} D_{b}^{\beta_{k s i}} p_{s} \in C^{1}([a, b]), a_{i}^{k s} \in C^{1}([a, b], \mathbb{R}), s=1, \ldots, r, i=1, \ldots, l$.

III. Operator of the third kind

$$
\begin{aligned}
& T^{k s}=T_{3}^{k s}:=a_{0}^{k s}(t)+a_{1}^{k s}(t)_{a}^{C} D_{t}^{\beta_{k s}}+a_{2}^{k s}(t)_{a}^{C} D_{t}^{1+\beta_{k s}}+\cdots+a_{l}^{k s}(t)_{a}^{C} D_{t}^{l-1+\beta_{k s}}, \quad 0<\beta_{k s} \leq 1, \\
& p_{s} \in C^{l}([a, b]) \text { and }{ }_{a}^{C} D_{t}^{1+\beta_{k s}} p_{s} \in C^{1}([a, b]), a_{i}^{k s} \in C^{1}([a, b], \mathbb{R}), s=1, \ldots, r .
\end{aligned}
$$

IV. Operator of the fourth kind

$$
\begin{aligned}
& T^{k s}=T_{4}^{k s}:=a_{0}^{k s}(t)+a_{1}^{k s}(t)_{t}^{C} D_{b}^{\beta_{k s}}+a_{1}^{k s}(t)_{t}^{C} D_{b}^{1+\beta_{k s}}+\cdots+a_{l}^{k s}(t)_{t}^{C} D_{b}^{l-1+\beta_{k s}}, \quad 0<\beta_{k s} \leq 1, \\
& p_{s} \in C^{l}([a, b]) \text { and }{ }_{t}^{C} D_{b}^{1+\beta_{k s}} p_{s} \in C^{1}([a, b]), a_{i}^{k s} \in C^{1}([a, b], \mathbb{R}), s=1, \ldots, r .
\end{aligned}
$$

Now we define the formal adjoint operator $\tilde{T}^{k s}$ of a fractional differential operator $T^{k s}$ similar in spirit to the classical case, by the integration by parts:

$$
\int_{a}^{b} q T^{k s}\left(p_{s}\right) d t=\int_{a}^{b} p_{s} \tilde{T}^{k s}(q) d t+[\cdot]_{t=a}^{t=b}
$$

where $[\cdot]_{t=a}^{t=b}$ represents the boundary terms. Therefore, the adjoints of $T_{i}^{k s}, i=1, \ldots, 4$, are given by expressions:

$$
\begin{gathered}
\int_{a}^{b} q T_{1}^{k s}\left(p_{s}\right) d t=\int_{a}^{b} p_{s} \tilde{T}_{1}^{k s}(q)=\int_{a}^{b} p_{s}\left(a_{0}^{k s} q+\sum_{i=1}^{l}{ }_{t} D_{b}^{\beta_{k s i}}\left(a_{i}^{k s} q\right)\right) d t+[\cdot]_{t=a}^{t=b}, \\
\int_{a}^{b} q T_{2}^{k s}\left(p_{s}\right) d t=\int_{a}^{b} p_{s} \tilde{T}_{2}^{k s}(q)=\int_{a}^{b} p_{s}\left(a_{0}^{k s} q+\sum_{i=1}^{l}{ }_{a} D_{t}^{\beta_{k s i}}\left(a_{i}^{k s} q\right)\right) d t+[\cdot]_{t=a}^{t=b}, \\
\int_{a}^{b} q T_{3}^{k s}\left(p_{s}\right) d t=\int_{a}^{b} p_{s} \tilde{T}_{3}^{k s}(q)=\int_{a}^{b} p_{s}\left(a_{0}^{k s} q+\sum_{i=0}^{l-1}{ }_{t} D_{b}^{i+\beta_{k s}}\left(a_{i+1}^{k s} q\right)\right) d t+[\cdot]_{t=a}^{t=b}, \\
\int_{a}^{b} q T_{4}^{k s}\left(p_{s}\right) d t=\int_{a}^{b} p_{s} \tilde{T}_{4}^{k s}(q)=\int_{a}^{b} p_{s}\left(a_{0}^{k s} q+\sum_{i=0}^{l-1}{ }_{a} D_{t}^{i+\beta_{k s}}\left(a_{i+1}^{k s} q\right)\right) d t+[\cdot]_{t=a}^{t=b} .
\end{gathered}
$$

Note that, if $p_{s}(a)=p_{s}(b)=0$, then boundary terms in (13) and (14) vanish. Similarly, if $p_{s}(a)=p_{s}^{(1)}(a)=\ldots=p_{s}^{(l-1)}(a)=0$ and $p_{s}(b)=p_{s}^{(1)}(b)=\ldots=p_{s}^{(l-1)}(b)=0$, then boundary terms in (15) and (16) vanish.

Now we define invariance. 
Definition 3.2. The functional (10) is invariant under transformations (111) if and only if for all $x \in C^{1}\left([a, b], \mathbb{R}^{n}\right)$ we have

$$
\int_{a}^{b} L\left(t, \bar{x}(t),{ }_{a}^{C} D_{t}^{\alpha} \bar{x}(t)\right) d t=\int_{a}^{b} L\left(t, x(t),{ }_{a}^{C} D_{t}^{\alpha} x(t)\right) d t .
$$

Theorem 3.3. If functional (10) is invariant under transformations (11), then there exist $r$ identities of the form

$$
\sum_{k=1}^{n} \tilde{T}^{k s}\left(E_{k}^{f}(L)\right)=0, \quad s=1, \ldots, r,
$$

where $\tilde{T}^{k s}$ is the adjoint of $T^{k s}$.

Proof. We give the proof only for the case $T^{k s}=T_{1}^{k s}$; other cases can be proved similarly. By Definition 3.2 we have

$$
\begin{aligned}
0=\int_{a}^{b} L\left(t, \bar{x}(t),{ }_{a}^{C} D_{t}^{\alpha} \bar{x}(t)\right) d t-\int_{a}^{b} L\left(t, x(t),{ }_{a}^{C} D_{t}^{\alpha} x(t)\right) d t \\
=\int_{a}^{b}\left(L\left(t, \bar{x}(t),{ }_{a}^{C} D_{t}^{\alpha} \bar{x}(t)\right)-L\left(t, x(t),{ }_{a}^{C} D_{t}^{\alpha} x(t)\right)\right) d t .
\end{aligned}
$$

Then, by the Taylor formula

$$
0=\sum_{k=1}^{n} \int_{a}^{b}\left[\frac{\partial L}{\partial x^{k}} T_{1}^{k s}\left(p_{s}\right)+\frac{\partial L}{\partial_{a}^{C} D_{t}^{\alpha_{k}} x^{k}}{ }^{C} D_{t}^{\alpha_{k}} T_{1}^{k s}\left(p_{s}\right)\right] d t,
$$

where $T_{1}^{k s}\left(p_{s}\right)=\sum_{s=1}^{r} T_{1}^{k s}\left(p_{s}\right)$. The second term in the integrand may be integrated by parts (see the first formula of (8) ):

$$
\int_{a}^{b} \frac{\partial L}{\partial_{a}^{C} D_{t}^{\alpha_{k}} x^{k}}{ }_{a}^{C} D_{t}^{\alpha_{k}} T_{1}^{k s}\left(p_{s}\right)=\left.T_{1}^{k s}\left(p_{s}\right)_{t} I_{b}^{1-\alpha_{k}} \frac{\partial L}{\partial_{a}^{C} D_{t}^{\alpha_{k}} x^{k}}\right|_{x=a} ^{x=b}+\int_{a}^{b} T_{1}^{k s}\left(p_{s}\right)_{t} D_{b}^{\alpha_{k}} \frac{\partial L}{\partial_{a}^{C} D_{t}^{\alpha_{k}} x^{k}} d t .
$$

Since $p_{s}$ are arbitrary, we may choose $p_{s}$ such that: $p_{s}(a)=p_{s}(b)=0$ and $\left.{ }_{a}^{C} D_{t}^{\beta_{k s i}} p_{s}(t)\right|_{t=b}=0$, $s=1, \ldots, r, i=1, \ldots, l$; and if $\beta_{k s i}=1$, then also $\left.{ }_{a}^{C} D_{t}^{\beta_{k s i}} p_{s}(t)\right|_{t=a}=0$. Therefore, the boundary term in (19) vanishes and substituting (19) into (18) we get

$$
0=\sum_{k=1}^{n} \int_{a}^{b}\left[\frac{\partial L}{\partial x^{k}}+{ }_{t} D_{b}^{\alpha_{k}} \frac{\partial L}{\partial_{a}^{C} D_{t}^{\alpha_{k}} x^{k}}\right] T_{1}^{k s}\left(p_{s}\right) d t .
$$

Using the definition of the adjoint operator $\tilde{T}_{1}^{k s}$ of a fractional differential operator $T_{1}^{k s}$, that is, equation (13), we get

$$
0=\sum_{k=1}^{n} \int_{a}^{b} \sum_{s=1}^{r} \tilde{T}_{1}^{k s}\left(E_{k}^{f}(L)\right) p_{s} d t+[\cdot]_{t=a}^{t=b} .
$$

Again appealing to the arbitrariness of $p_{s}$ we can force the boundary term to vanish, and finally by the fundamental lemma of calculus of variations we conclude that

$$
\sum_{k=1}^{n} \tilde{T}_{1}^{k s}\left(E_{k}^{f}(L)\right)=0, \quad s=1, \ldots, r .
$$


Remark 2. Notice that if we put $\beta_{k s}=1$ in transformations of the third or the fourth kind, then we obtain infinitesimal transformations:

$$
\left\{\begin{array}{l}
\bar{t}=t \\
\bar{x}^{k}(t)=x^{k}+B^{k s}\left(p_{s}\right)+\ldots
\end{array}\right.
$$

where

$$
B^{k s}=b_{0}^{k s}(t)+b_{1}^{k s}(t) \frac{d}{d t}+b_{2}^{k s}(t) \frac{d^{2}}{d t^{2}}+\cdots+b_{l}^{k s}(t) \frac{d^{l}}{d t^{l}}, \quad k=1, \ldots, n .
$$

In this case the adjoint operator $\tilde{B}^{k s}$ of the differential operator $B^{k s}$ is given by

$$
\tilde{B}^{k s}(q)=b_{0}^{k s} q+\sum_{i=1}^{l}(-1)^{i} \frac{d^{i}}{d t^{i}}\left(b_{i}^{k s} q\right), \quad k=1, \ldots, n
$$

and the identities (17) take the form

$$
\sum_{k=1}^{n} b_{0}^{k s}\left(E_{k}(L)\right)+\sum_{k=1}^{n} \sum_{i=1}^{l}(-1)^{i} \frac{d^{i}}{d t^{i}}\left(b_{i}^{k s} E_{k}(L)\right)=0, \quad s=1, \ldots, r,
$$

which are exactly the Noether identities (see Brading (2002); Logan (1974)).

Remark 3. The fractional differential operators $T_{1}^{k s}, T_{2}^{k s}, T_{3}^{k s}$ and $T_{4}^{k s}$ can of course be combined, that is, we can consider infinitesimal transformations that depend upon arbitrary functions and their fractional derivatives in the sense of Caputo: left and right with various orders.

\subsection{Multiple Integral Case}

Consider a system characterized by a set of functions

$$
u^{j}\left(t, x_{1}, \ldots, x_{m}\right), \quad j=1, \ldots, n,
$$

depending on time $t$ and the space coordinates $x_{1}, \ldots, x_{m}$. We can simplify the notation by interpreting (20) as a vector function $u=\left(u^{1}, \ldots, u^{n}\right)$ and writing $t=x_{0}, x=\left(x_{0}, x_{1}, \ldots, x_{m}\right)$, $d x=d x_{0} d x_{1} \cdots d x_{m}$. Then (20) becomes simply $u(x)$ and is called a vector field. Define the action functional in the form

$$
\mathcal{J}(u)=\int_{\Omega} \mathcal{L}\left(x, u,{ }^{C} \nabla^{\alpha} u\right) d x
$$

where $\Omega=R \times\left[a_{0}, b_{0}\right], R=\left[a_{1}, b_{1}\right] \times \ldots \times\left[a_{m}, b_{m}\right]$, and ${ }^{C} \nabla^{\alpha}$ is the operator

$$
\left(\begin{array}{l}
C \\
a_{0}
\end{array} D_{x_{0}}^{\alpha_{0}},{ }_{a_{1}}^{C} D_{x_{1}}^{\alpha_{1}}, \cdots,{ }_{a_{m}}^{C} D_{x_{m}}^{\alpha_{m}}\right),
$$

where $\alpha=\left(\alpha_{0}, \alpha_{1}, \ldots, \alpha_{m}\right), 0<\alpha_{i} \leq 1, i=0, \ldots, m$. The function $\mathcal{L}\left(x, u,{ }^{C} \nabla^{\alpha} u\right)$ is called the fractional Lagrangian density of the field. We assume that:

(i) $u^{j} \in C^{1}(\Omega, \mathbb{R}), j=1, \ldots, n$;

(ii) $\mathcal{L} \in C^{1}\left(\mathbb{R}^{m} \times \mathbb{R}^{n} \times \mathbb{R}^{n(m+1)} ; \mathbb{R}\right)$; 
(iii) $x \rightarrow \frac{\partial \mathcal{L}}{\partial_{a_{i}}^{C} D_{x_{i}}^{\alpha_{i}} u^{j}}$ for every $u^{j} \in C^{1}(\Omega, \mathbb{R})$ are $C^{1}$ functions, $j=1, \ldots, n, i=0, \ldots, m$.

Define the admissible set of functions $A(\Omega)$ by

$$
A(\Omega):=\left\{u: \Omega \rightarrow \mathbb{R}^{n}: u(x)=\varphi(x) \text { for } \quad x \in \partial \Omega\right\},
$$

where $\varphi: \partial \Omega \rightarrow \mathbb{R}^{n}$ is a given function.

Applying the principle of stationary action to (21) we obtain the multidimensional fractional Euler-Lagrange equations for the field.

Theorem 3.4. [cf. Cresson (2007)] A necessary condition for the function $u \in A(\Omega)$ to provide an extremum for the action functional (21) it that its components satisfy the $n$ multidimensional fractional Euler-Lagrange equations:

$$
\frac{\partial \mathcal{L}}{\partial u^{j}}+\sum_{i=0}^{m} x_{i} D_{b_{i}}^{\alpha_{i}} \frac{\partial \mathcal{L}}{\partial_{a_{i}}^{C} D_{x_{i}}^{\alpha_{i}} u^{j}}=0, \quad j=1, \ldots, n .
$$

As before we define

$$
E_{j}^{f}(\mathcal{L}):=\frac{\partial \mathcal{L}}{\partial u^{j}}+\sum_{i=0}^{n} x_{i} D_{b_{i}}^{\alpha_{i}} \frac{\partial \mathcal{L}}{\partial_{a_{i}}^{C} D_{x_{i}}^{\alpha_{i}} u^{j}},
$$

which are called the fractional Lagrange expressions.

We shall study infinitesimal transformations that depend upon arbitrary functions of independent variables and their partial fractional derivatives in the sense of Caputo. Let

$$
\left\{\begin{array}{l}
\bar{x}=x, \\
\bar{u}^{j}(x)=u^{j}(x)+T^{j 1}\left(p_{1}(x)\right)+\cdots+T^{j r}\left(p_{r}(x)\right), \quad j=1, \ldots, n,
\end{array}\right.
$$

where $T^{j s}$ are linear fractional differential operators and $p_{s}, s=1, \ldots, r$ are the $r$ arbitrary, independent $C^{1}$ functions defined on $\Omega$. Then, we consider two types of fractional differential operators:

I. Operator of the first kind

$$
T^{j s}=T_{1}^{j s}:=c^{j s}(x)+\sum_{i=0}^{m} c_{i}^{j s}(x)_{a_{i}}^{C} D_{x_{i}}^{\beta_{j s i}}, \quad 0<\beta_{j s i} \leq 1,
$$

and ${ }_{a_{i}}^{C} D_{x_{i}}^{\beta_{j s i}} p_{s}, c^{j s}, c_{i}^{j s}$ are $C^{1}$ functions defined on $\Omega, s=1, \ldots, r, i=1, \ldots, m$.

II. Operator of the second kind

$$
T^{j s}=T_{1}^{j s}:=c^{j s}(x)+\sum_{i=0}^{m} c_{i}^{j s}(x)_{x_{i}}^{C} D_{b_{i}}^{\beta_{j s i}}, \quad 0<\beta_{j s i} \leq 1,
$$

and ${ }_{x_{i}}^{C} D_{b_{i}}^{\beta_{j s i}} p, c^{j s}, c_{i}^{j s}$ are $C^{1}$ functions defined on $\Omega, s=1, \ldots, r, i=1, \ldots, m$.

We define invariance similarly to the one-dimensional case. 
Definition 3.5. The functional (21) is invariant under transformations (22) if and only if for all $u \in C^{1}\left(\Omega, \mathbb{R}^{n}\right)$ we have

$$
\int_{\Omega} \mathcal{L}\left(x, \bar{u},{ }^{C} \nabla^{\alpha} \bar{u}\right) d x=\int_{\Omega} \mathcal{L}\left(x, u,{ }^{C} \nabla^{\alpha} u\right) d x
$$

Theorem 3.6. If functional (21) is invariant under transformations (22), then there exist $r$ identities of the form

$$
\sum_{j=1}^{n} \tilde{T}^{j s}\left(E_{j}^{f}(\mathcal{L})\right)=0, \quad s=1, \ldots, r,
$$

where $\tilde{T}^{j s}$ is the adjoint of $T^{j s}$.

Proof. We give the proof only for the case $T^{j s}=T_{1}^{j s}$; the other case can be proved similarly. By Definition 3.5 we have

$$
0=\int_{\Omega} \mathcal{L}\left(x, \bar{u},{ }^{C} \nabla^{\alpha} \bar{u}\right) d x-\int_{\Omega} \mathcal{L}\left(x, u,{ }^{C} \nabla^{\alpha} u\right) d x=\int_{\Omega}\left(\mathcal{L}\left(x, \bar{u},{ }^{C} \nabla^{\alpha} \bar{u}\right)-\mathcal{L}\left(x, u,{ }^{C} \nabla^{\alpha} u\right)\right) d x .
$$

Then, by the Taylor formula

$$
0=\sum_{j=1}^{n} \int_{\Omega}\left(\frac{\partial \mathcal{L}}{\partial u^{j}} T_{1}^{j s}\left(p_{s}\right)+\sum_{i=0}^{m} \frac{\partial \mathcal{L}}{\partial_{a_{i}}^{C} D_{x_{i}}^{\alpha_{i}} u^{j}}{ }^{C} D_{i} D_{x_{i}}^{\alpha_{i}} T_{1}^{j s}\left(p_{s}\right)\right) d x
$$

where $T_{1}^{j s}\left(p_{s}\right)=\sum_{s=1}^{r} T_{1}^{j s}\left(p_{s}\right)$. The Fubini theorem allows us to rewrite integrals as the iterated integrals so that we can use the integration by parts formula (8):

$$
\int_{\Omega} \sum_{i=0}^{m} \frac{\partial \mathcal{L}}{\partial_{a_{i}}^{C} D_{x_{i}}^{\alpha_{i} u^{j}}{ }^{C}{ }_{i}} D_{x_{i}}^{\alpha_{i}} T_{1}^{j s}\left(p_{s}\right) d x=\int_{\Omega} \sum_{i=0}^{m}{ }_{x_{i}} D_{b_{i}}^{\alpha_{i}} \frac{\partial \mathcal{L}}{\partial_{a_{i}}^{C} D_{x_{i}}^{\alpha_{i} u^{j}}} T_{1}^{j s}\left(p_{s}\right) d x+\left.[\cdot]\right|_{\partial \Omega}, \quad j=1, \ldots, m,
$$

where $\left.[\cdot]\right|_{\partial \Omega}$ represent the boundary terms $-m$-volumes integrals. Since $p_{s}$ are arbitrary, we may choose $p_{s}$ such that: $\left.p_{s}(x)\right|_{\partial \Omega}=0$ and $\left.{ }_{a_{i}}^{C} D_{x_{i}}^{\beta_{j s i}} p_{s}(t)\right|_{\partial \Omega}=0, s=1, \ldots, r, i=1, \ldots, l$. Therefore, the boundary term in (24) vanishes and substituting (24) into (23) we get

$$
0=\sum_{j=1}^{n} \int_{\Omega}\left(\frac{\partial \mathcal{L}}{\partial u^{j}}+\sum_{i=0}^{m} x_{i} D_{b_{i}}^{\alpha_{i}} \frac{\partial \mathcal{L}}{\partial_{a_{i}}^{C} D_{x_{i}}^{\alpha_{i} u^{j}}}\right) T_{1}^{j s}\left(p_{s}\right) d x
$$

Now we proceed as in the one-dimensional case and define the adjoint operator $\tilde{T}_{1}^{j s}$ of a fractional differential operator $T_{1}^{j s}$ by

$$
\int_{\Omega} q(x) T_{1}^{j s}\left(p_{s}(x)\right) d x=\int_{\Omega} p_{s}(x) \tilde{T}_{1}^{j s}(q(x)) d x+\left.[\cdot]\right|_{\partial \Omega}, \quad j=1, \ldots, n, \quad s=1, \ldots, r,
$$

where we use the Fubini theorem. Again appealing to the arbitrariness of $p_{s}$ we can force the boundary term to vanish (by putting $\left.p_{s}(x)\right|_{\partial \Omega}=0$ ). Therefore,

$$
0=\sum_{j=1}^{n} \int_{\Omega} \sum_{s=1}^{r} \tilde{T}_{1}^{j s}\left(\frac{\partial \mathcal{L}}{\partial u^{j}}+\sum_{i=0}^{m} x_{i} D_{b_{i}}^{\alpha_{i}} \frac{\partial \mathcal{L}}{\partial_{a_{i}}^{C} D_{x_{i}}^{\alpha_{i}} u^{j}}\right) p_{s} d x
$$


Finally by the fundamental lemma of calculus of variations we conclude that

$$
\sum_{j=1}^{n} \tilde{T}_{1}^{j s}\left(E_{j}^{f}(\mathcal{L})\right)=0, \quad s=1, \ldots, r .
$$

Remark 4. The adjoints of $T_{i}^{j s}, i=1,2$, are given by expressions:

$$
\begin{aligned}
& \tilde{T}_{1}^{j s}(q)=c^{j s} q+\sum_{i=0}^{m} x_{i} D_{b_{i}}^{\beta_{j s i}}\left(c_{i}^{j s} q\right), \quad j=1, \ldots, n, \\
& \tilde{T}_{2}^{j s}(q)=c^{j s} q+\sum_{i=0}^{m} a_{i} D_{x_{i}}^{\beta_{j s i}}\left(c_{i}^{j s} q\right), \quad j=1, \ldots, n .
\end{aligned}
$$

Remark 5. The fractional differential operators $T_{1}^{j s}$ and $T_{2}^{j s}$ can of course be combined, that is, we can consider infinitesimal transformations that depend upon arbitrary functions and their partial fractional derivatives in the sense of Caputo: left and right with various orders.

\section{Example}

In order to illustrate our result we will use the Lagrangian density for the electromagnetic field (see Gelfand and Fomin (2000)):

$$
\mathcal{L}=\frac{1}{8 \pi}\left(\mathbf{E}^{2}-\mathbf{H}^{2}\right)
$$

where $\mathbf{E}$ and $\mathbf{H}$ are the electric field vector and the magnetic field vector, respectively. Following (Baleanu et al. (2009)) we shall generalize (25) to the fractional Lagrangian density by changing classical partial derivatives by fractional. Let $x=\left(x_{0}, x_{1}, x_{2}, x_{3}\right) \in \Omega$; and $\mathbf{A}(x)=\left(A_{1}(x), A_{2}(x), A_{3}(x)\right), A_{0}(x)$ be a vector potential and a scalar potential, respectively. They are defined by setting

$$
\mathbf{E}=\operatorname{grad}^{\left(\alpha_{1}, \alpha_{2}, \alpha_{3}\right)} A_{0}-{ }_{a_{0}}^{C} D_{x_{0}}^{\alpha_{0}} \mathbf{A}, \quad \mathbf{H}=\operatorname{curl} \mathbf{A}, \quad 0<\alpha_{i} \leq 1, \quad i=0, \ldots, 3,
$$

where

$$
\begin{gathered}
\operatorname{grad}^{\left(\alpha_{1}, \alpha_{2}, \alpha_{3}\right)} A_{0}=\mathbf{i}_{a_{1}}^{C} D_{x_{1}}^{\alpha_{1}} A_{0}+\mathbf{j}_{a_{2}}^{C} D_{x_{2}}^{\alpha_{2}} A_{0}+\mathbf{k}_{a_{3}}^{C} D_{x_{3}}^{\alpha_{3}} A_{0}, \\
{ }_{a_{0}}^{C} D_{x_{0}}^{\alpha_{0}} \mathbf{A}=\mathbf{i}_{a_{0}}^{C} D_{x_{0}}^{\alpha_{0}} A_{1}+\mathbf{j}_{a_{0}}^{C} D_{x_{0}}^{\alpha_{0}} A_{2}+\mathbf{k}_{a_{0}}^{C} D_{x_{0}}^{\alpha_{0}} A_{3}, \\
\operatorname{curl} \mathbf{A}=\mathbf{i}\left({ }_{a_{2}}^{C} D_{x_{2}}^{\alpha_{2}} A_{3}-{ }_{a_{3}}^{C} D_{x_{3}}^{\alpha_{3}} A_{2}\right)+\mathbf{j}\left({ }_{a_{3}}^{C} D_{x_{3}}^{\alpha_{3}} A_{1}-{ }_{a_{1}}^{C} D_{x_{1}}^{\alpha_{1}} A_{3}\right)+\mathbf{k}\left({ }_{a_{1}}^{C} D_{x_{1}}^{\alpha_{1}} A_{2}-{ }_{a_{2}}^{C} D_{x_{2}}^{\alpha_{2}} A_{1}\right) .
\end{gathered}
$$

Replacing $\mathbf{E}$ and $\mathbf{H}$ in (25) by their expressions (26) we obtain the fractional Lagrangian density

$$
\mathcal{L}=\frac{1}{8 \pi}\left[\left(\operatorname{grad}^{\left(\alpha_{1}, \alpha_{2}, \alpha_{3}\right)} A_{0}-{ }_{a_{0}}^{C} D_{x_{0}}^{\alpha_{0}} \mathbf{A}\right)^{2}-(\operatorname{curl} \mathbf{A})^{2}\right] .
$$

Note that, similarly to the integer case, the potential $\left(A_{0}, \mathbf{A}\right)$ is not uniquely determined by the vectors $\mathbf{E}$ and $\mathbf{H}$. Namely, $\mathbf{E}$ and $\mathbf{H}$ do not change if we make a gauge transformation:

$$
\tilde{A}_{j}(x)=A_{j}(x)+{ }_{a_{j}}^{C} D_{x_{j}}^{\alpha_{j}} f(x), \quad j=0, \ldots, 3,
$$


where $f: \Omega \rightarrow \mathbb{R}$ is an arbitrary function of class $C^{2}$ in all of its argument. Therefore, the Lagrangian density (27), and hence the action functional, is invariant under transformation (28). By Theorem (3.6), we conclude that

$$
\sum_{j=0}^{3}{ }_{x_{j}} D_{b_{j}}^{\alpha_{j}}\left(E_{j}^{f}(\mathcal{L})\right)=0
$$

where $E_{j}^{f}(\mathcal{L})$ are Lagrange expressions corresponding to (27). Equations $E_{j}^{f}(\mathcal{L})=0$ do not uniquely determine the potential $\left(A_{0}, \mathbf{A}\right)$ and to avoid this lack of uniqueness, the fractional Lorentz condition can be imposed on $\left(A_{0}, \mathbf{A}\right)$.

\section{Acknowledgments}

The author is supported by the Białystok University of Technology Grant S/WI/2/2011 and by the European Union Human Capital Programme: Podniesienie potencjału uczelni wyzszych jako czynnik rozwoju gospodarki opartej na wiedzy. The author is grateful to two anonymous reviewers for their comments.

\section{References}

Agrawal OP (2007) Generalized Euler-Lagrange equations and transversality conditions for FVPs in terms of the Caputo derivative. Journal of Vibration and Control 13(9-10): 12171237.

Agrawal OP, Muslih SI and Baleanu D (2011) Generalized variational calculus in terms of multi-parameters fractional derivatives. Communications in Nonlinear Science and Numerical Simulation 16(12): 4756-4767.

Almeida R and Torres DFM (2009) Calculus of variations with fractional derivatives and fractional integrals. Applied Mathematics Letters 22(12): 1816-1820. arXiv:0907.1024

Almeida R, Malinowska AB and Torres DFM (2010) A fractional calculus of variations for multiple integrals with application to vibrating string. Journal of Mathematical Physics 51(3): 033503, 12 pp. arXiv:1001.2722 v1

Almeida R, Pooseh S and Torres DFM (2012), Fractional variational problems depending on indefinite integrals. Nonlinear Analysis. Theory, Methods \& Applications 75(3): 1009-1025. arXiv:1102.3360

Atanacković TM, Konjik S and Pilipović S (2008) Variational problems with fractional derivatives: Euler-Lagrange equations. Journal of Physics A 41(9): 095201, 12 pp.

Atanacković TM, Konjik S Pilipović S and Simic S (2009) Variational problems with fractional derivatives: Invariance conditions and Noether's theorem. Nonlinear Analysis. Theory, Methods \& Applications 71(5-6): 1504-1517.

Baleanu D and Avkar T (2004) Lagrangians with linear velocities within Riemann-Liouville fractional derivatives. Nuovo Cimento 119: 7379. 
Baleanu D and Muslih S (2005) Lagrangian formulation of classical fields within RiemannLiouville fractional derivatives. Physica Scripta 72: 119-121.

Baleanu D and Agrawal OP (2006) Fractional Hamilton formalism within Caputo's derivative. Czechoslovak Journal of Physics 56(10-11): 1087-1092.

Baleanu D (2008) Fractional constrained systems and Caputo derivatives. Journal of Computational and Nonlinear Dynamics 3:021102.

Baleanu D, Golmankhaneh AK Golmankhaneh AK and Baleanu MC (2009) Fractional electromagnetic equations using fractional forms. International Journal of Theoretical Physics 48: 3114-3123.

Baleanu D, Diethelm K, Scalas E and Trujillo JJ (2011) Fractional calculus: models and numerical methods. World Scientific.

Brading K (2002) Which Symmetry Noether, Weyl and conservation of electric charge. Studies in History and Philosophy of Science. Part B. Studies in History and Philosophy of Modern Physics 33: 3-22.

Carinena JF, Lazaro-Cami JA and Martinez E (2005) On second Noether's theorem and gauge symmetries in Mechanics. International Journal of Geometric Methods in Modern Physics 3(3): 1-14.

Cresson J (2007) Fractional embedding of differential operators and Lagrangian systems. Journal of Mathematical Physics 48(3): 033504, 34 pp.

El-Nabulsi RA and Torres DFM (2007) Necessary optimality conditions for fractional actionlike integrals of variational calculus with Riemann-Liouville derivatives of order $(\alpha, \beta)$. Mathematical Methods in the Applied Sciences 30(15): 1931-1939.

El-Nabulsi RA (2011) A periodic functional approach to the calculus of variations and the problem of time-dependent damped harmonic oscillators. Applied Mathematics Letters 2: $1647-1653$.

Fonseca Ferreira MN, Duarte FB, Lima MFM et al. (2008) Application of fractional calculus in the dynamical analysis and control of mechanical manipulators. Fractional Calculus \& Applied Analysis 11(1): 91-113.

Frederico GSF and Torres DFM (2007) A formulation of Noether's theorem for fractional problems of the calculus of variations. Journal of Applied Mathematical Analysis and Applications 334(2): 834-846. arXiv:math/0701187v1

Frederico GSF and Torres DFM (2008) Fractional conservation laws in optimal control theory. Nonlinear Dynamics 53(3): 215-222. arXiv:0711.0609

Frederico GSF and Torres DFM (2010) Fractional Noether's theorem in the Riesz-Caputo sense. Applied Mathematics and Computation 217(3): 1023-1033. arXiv:1001.4507

Gelfand IM and Fomin SV (2000) Calculus of variations. Dover Publications, Mineola, NY.

Herrmann R (2008) Gauge invariance in fractional field theories. Physics Letters. A 372: $5515-5522$. 
Herzallah Mohamed A. E.; Baleanu Dumitru (2011) Fractional-order variational valculus with generalized boundary conditions. Advances in Difference equations, Article ID 357580, 9 pages, doi:10.1155/2011/357580.

Hilfer R (2000) Applications of fractional calculus in physics. World Scientific Publishing, River Edge, NJ.

Hydon PE and Mansfield EL (2011) Extensions of Noethers Second Theorem: from continuous to discrete systems. Proceedings the Royal Society A 00: (accessed June 22).

Jumarie G (2007) Fractional Hamilton-Jacobi equation for the optimal control of nonrandom fractional dynamics with fractional cost function. Journal of Applied Mathematics and Computing 23(1-2): 215-228.

Kosmann-Schwarzbach Y (2010) The Noether theorems. Invariance and conservation laws in the twentieth century. New York, Springer.

Kilbas AA, Srivastava HM and Trujillo JJ (2006) Theory and applications of fractional differential equations. Elsevier, Amsterdam.

Klimek M (2009) On solutions of linear fractional differential equations of a variational type. The Publishing Office of Czestochowa University of Technology, Czestochowa.

Logan JD (1974) On variational problems which admit an infinite continuous group, Yokohama Mathematical Journal 22: 31-42.

Logan JD (1977) Invariant variational principles. Academic Press [Harcourt Brace Jovanovich Publishers], New York.

Love ER and Young LC (1938) On fractional integration by parts, Proceedings of the London Mathematical Society 44: 1-35.

Malinowska AB and Torres DFM (2010) Generalized natural boundary conditions for fractional variational problems in terms of the Caputo derivative. Computers \& Mathematics with Applications 59(9): 3110-3116. arXiv:1002.3790v1

Malinowska AB (2012) Fractional variational calculus for non-differentiable functions. In Baleanu D, Tenreiro Machado JA and Luo ACJ (eds.) Fractional Dynamics and Control. Springer Science+Business Media, Part 2, 97-108. arXiv:1201.6640v1

Malinowska AB and Torres DFM (2011) Fractional calculus of variations for a combined Caputo derivative, Fractional Calculus \& Applied Analysis 14(4): 523-537. arXiv:1109.4664v1

Podlubny I (1999) Fractional differential equations. Academic Press, San Diego, CA.

Riewe F (1996) Nonconservative Lagrangian and Hamiltonian mechanics. Physical Review E (3) 53(2): 1890-1899.

Riewe F (1997) Mechanics with fractional derivatives. Physical Review E (3) 55(3): 35813592.

Samko SG, Kilbas AA and Marichev OI (1993) Fractional integrals and derivatives. Translated from the 1987 Russian original, Gordon and Breach, Yverdon. 
Tenreiro Machado AJ, Kiryakova V and Mainardi F (2010) A poster about the old history of fractional calculus. Fractional Calculus \& Applied Analysis 13: 447-454.

Tenreiro Machado AJ, Kiryakova V and Mainardi F (2011) Recent history of fractional calculus. Communications in Nonlinear Science and Numerical Simulation 16: 1140-1153.

Torres DFM (2003) Gauge symmetries and Noether currents in optimal control. Applied Mathematics E-Notes 3: 49-57. arXiv:math/0301116v1

Tarasov VE (2006) Fractional variations for dynamical systems: Hamilton and Lagrange approaches. Journal of Physics A 39(26): 8409-8425.

Tarasov VE (2008) Fractional vector calculus and fractional Maxwell's equations, Annals of Physics 323(11): 2756-2778. 